\title{
Ripretinib-a new star in the firmament
}

\author{
Attila Kollár \\ Department of Medical Oncology, Inselspital, Bern University Hospital, University of Bern, Switzerland \\ Correspondence to: Dr. Attila Kollár, MD. Department of Medical Oncology, Inselspital, Bern University Hospital, University of Bern, Lory-Spital, \\ CH-3010 Bern, Switzerland. Email: attila.kollar@insel.ch.
}

Received: 25 February 2021. Accepted: 30 April 2021; Published: 30 July 2021.

doi: $10.21037 /$ gist-21-3

View this article at: https://dx.doi.org/10.21037/gist-21-3

\section{Introduction}

Gastrointestinal stromal tumor (GIST) represents the most common mesenchymal tumor in the gastrointestinal tract, representing $1-3 \%$ of gastrointestinal malignancies (1). With an annual incidence of approximately 15 per million per year GIST belongs to the orphan disease group (2). The interstitial cells of Cajal, which are part of the autonomic nervous system of the intestine, are the cell of origin. In the adult population, the majority of GISTs occur in the stomach and in the small intestine. However, it may develop at any site or even outside of the gastrointestinal tract too (3).

In the localized stage tumor resection is the treatment of choice. The indication for adjuvant imatinib depends on an estimation of the risk of recurrence, which is based upon different risk stratification tools including tumor-associated risk factors like tumor size, number of mitoses, primary site, and the presence or absence of tumor rupture and importantly mutational status $(4,5)$.

Until recently, imatinib, sunitinib and regorafenib reflected the current treatment armamentarium in the metastatic disease setting in the order listed (6-8). The initially poor prognosis of this rather chemotherapyresistant neoplasia was dramatically improved with the introduction of those molecularly targeted agents, which blocks signaling via c-kit (KIT) or platelet-derived growth factor receptor alpha (PDGFRA)-related signaling pathways. The first impressive step forward represented the approval of imatinib by the US Food and Drug Administration (FDA) in 2002. Treatment with imatinib was associated with a partial response rate of more than $50 \%$ and has increased the median survival from an average of 18 months in the past to 57 months (9). Sunitinib is another orally administered multi-targeted receptor tyrosine kinase inhibitor. The evidence for its benefit comes from an international phase III trial comparing its efficacy with placebo. This landmark trial included 312 patients with imatinib-refractory disease. Despite a low objective response rate in the sunitinib group (7\%), the median time to tumor progression, the primary endpoint, could be significantly improved from 6 to 27 weeks, respectively (7). The tyrosine kinase inhibitor regorafenib gained approval by FDA based on the results of the GRID Phase III trial. In that study, patients with histologically confirmed, metastatic or unresectable GIST, with failure of at least previous imatinib and sunitinib were randomized in a 2:1 ratio, to receive either oral regorafenib $160 \mathrm{mg}$ daily or placebo, plus best supportive care (BSC) in both groups. Median progressionfree-survival (PFS) per independent blinded central review (primary endpoint) was 4.8 months (IQR 1.4-9.2) for regorafenib and 0.9 months $(0.9-1.8)$ for placebo [hazard ratio (HR) 0.27, 95\% CI: 0.19-0.39; $\mathrm{P}<0.0001$ ] (Table 1) (8).

After failing the approved drugs, GIST patients are usually treated within clinical trials or, if available, with other off-label tyrosine kinase inhibitors (TKIs i.e., pazopanib, nilotinib) $(10,11)$. There are limited data to support that rechallenge with prior failed treatment options may offer a clinically relevant benefit. A Korean, singlecenter, randomized, placebo-controlled study ('RIGHT Trial') demonstrated that a rechallenge with imatinib after imatinib and sunitinib failure provides a statistically significant, but clinically small benefit to some patients compared to placebo (median PFS of 1.8 and 0.9 months, respectively) (12).

For patients who have progressed on the approved agents, progression of KIT-driven tumors is primarily based on development of further KIT resistance mutations that are highly heterogeneous. Data from studies show that approved TKIs do not fully cover these secondary resistance 
Table 1 Comparison of treatment lines

\begin{tabular}{|c|c|c|c|c|}
\hline Line of therapy & 1st & 2nd & $3 r d$ & 4th \\
\hline Median PFS & $\begin{array}{l}\text { I,400 mg: } 20.4 \text { mo; } \\
\text { I } 800 \text { mg: } 24 \text { mo; } P=0.18\end{array}$ & $\begin{array}{l}\text { S: } 5.6 \text { mo; } \\
\text { Placebo: } 1.4 \text { mo; } P<0.0001\end{array}$ & $\begin{array}{l}\text { Re: } 4.8 \text { mo; } \\
\text { Placebo: } 0.9 \text { mo; } P<0.0001\end{array}$ & $\begin{array}{l}\text { Ri: } 6.3 \text { mo; } \\
\text { Placebo: } 1.0 \text { mo; } P<0.0001\end{array}$ \\
\hline $\begin{array}{l}\text { Overall response } \\
\text { rate }(\mathrm{CR}+\mathrm{PR})\end{array}$ & $\begin{array}{l}\text { I,400 mg: 51.0\%; } \\
\text { I,800 mg: 56.7\%; P=0.08 }\end{array}$ & $\begin{array}{l}\text { S: } 6.8 \% \text {; Placebo: } 0 \% \text {; } \\
P<0.006\end{array}$ & $\begin{array}{l}\text { Re: } 4.5 \% \text {; Placebo: } 1.5 \% ; \\
P=N R\end{array}$ & $\begin{array}{l}\text { Ri: 9\%; Placebo: 0\%; } \\
P<0.0504\end{array}$ \\
\hline
\end{tabular}

PFS, progression-free survival; OS, overall survival; mo, months; CR, complete response; PR, partial response; NR, not reported; NA, not applicable.

mutations (13). Hence, the use of serially applied TKIs, like ripretinib, for advanced GIST represents a common treatment strategy.

\section{Mechanism of action}

KIT and PDGFRa are proto-oncogene receptor tyrosine kinase receptors that play an important role in the regulation of tumor cell proliferation. In GIST, they are upregulated or mutated and their blockage may therefore inhibit tumor cell growth.

Ripretinib (DCC-2618) is an orally bioavailable tyrosine kinase inhibitor. It boasts a unique dual mechanism of action. In addition to binding to the kinase switch pocket it targets the activation loop as well, thereby turning off the kinase and its ability to cause dysregulated cell growth. This is in contrast to other agents used for the treatment of patients with GIST, which bind the kinase at the adenosine triphosphate (ATP)-binding site, hence, try to prevent ATP from binding and leading to activation of the kinase. Thereby ripretinib succeeds to inhibit the proliferation of wild-type as well as mutant GIST forms (exons 9, 11, 13, 14, 17 and 18 for KIT mutations and exons 12, 14 and 18 for PDGFRA mutations) $(14,15)$. Additionally, tumor cell growth may be reduced by ripretinib blocking several other kinases, including vascular endothelial growth factor receptor type 2 (VEGFR2), angiopoietin-1 receptor (TIE2), PDGFR-beta and macrophage colony-stimulating factor 1 receptor (CSF1R) (16).

Ripretinib is absorbed in the gastrointestinal tract and bound in over $99 \%$ to albumin and alpha-1 acid glycoprotein. It represents a prodrug which is metabolized by the CYP3A subfamily of enzymes with contributions from CYP2D6 and CYP2E1 to its active metabolite, DP-
5439. One third is excreted in the feces and only a marginal amount in the urine. The average half-life of ripretinib is 14.8 hours. No clinically significant differences in the pharmacokinetics were observed based on age, sex, race, body weight, primary tumor (GIST or other solid tumors), prior gastrectomy, mild to moderate renal impairment (CLcr 30 to $<90 \mathrm{~mL} / \mathrm{min}$ estimated by Cockcroft-Gault), and mild hepatic impairment (total bilirubin $\leq \mathrm{ULN}$ and AST $>\mathrm{ULN}$ or total bilirubin 1 to $1.5 \times$ ULN and AST any). The effects of severe renal impairment or moderate to severe hepatic impairment have not been studied (17).

Notably, co-administration of ripretinib with a strong CYP3A inhibitor may increase the exposure of ripretinib and its active metabolite, which may increase the risk of adverse events (17).

\section{Clinical trials}

\section{Efficacy}

Based on promising preclinical data the safety of ripretinib was investigated in a dose escalation and expansion phase 1 trial. George at al. reported on the results of this dose-finding study. Although no dose-limiting toxicity has been experienced using doses up to $300 \mathrm{mg}$ per day, $150 \mathrm{mg}$ once daily (QD) was chosen for further evaluation based on available pharmacological, toxicity and efficacy data. These initial results demonstrated an impressive progression free survival in a meaningful subset of patients across all treatment lines (18) and therefore, paving the way for further investigation.

Recently, Blay et al. published the results of the Invictus trial (19). In this international, multi-center, randomized (2:1), double-blind, placebo-controlled, phase 3 study the efficacy and safety of ripretinib plus BSC, was compared with placebo plus BSC in patients with progression on at 
least imatinib, sunitinib, and regorafenib or documented intolerance to any of these treatments for previously treated, advanced GIST. Randomization was stratified by prior lines of therapy ( 3 versus $\geq 4$ ) and Eastern Cooperative Oncology Group (ECOG) performance status (0 versus 1 or 2). Patients received Ripretinib $150 \mathrm{mg}$ once daily QD or placebo until disease progression or unacceptable toxicity. Patients randomized to receive placebo could be treated with ripretinib at the time of disease progression. The primary endpoint was median PFS based on disease assessment by blinded independent central review using modified RECIST 1.1 criteria. 129 patients were randomized. Median age was 60 years (range, 29 to 83 years) and $92 \%$ had an ECOG performance status of 0 or $1.63 \%$ of patients received three prior therapies and $37 \%$ received 4 or more prior therapies. $66 \%$ of patients randomized to placebo switched to ripretinib after disease progression. At a median follow-up of 6.3 months, median PFS was 6.3 months (95\% CI: 4.6-6.9 months) with ripretinib compared with 1 months (0.9-1.7 months) with placebo (hazard ratio 0.15, 95\% CI: 0.09-0.25; $\mathrm{P}<0.0001$ ) meeting the primary endpoint. The confirmed objective response rate according to mRECIST criteria 1.1 was $9 \%$ in the ripretinib arm and $0 \%$ in the placebo group. Notably, no complete responses could be observed. Although the statistical design did not allow to formally test the OS, a secondary endpoint, median OS in the ripretinib and placebo arm was 15.1 months (95\% CI: $12.3-15.1$ months) and 6.6 months (4.1-11.6 months) (HR 0.36, 95\% CI: 0.21-0.62), respectively. Based on those results FDA approved Deciphera pharmaceuticals' ripretinib for fourthline therapy of advanced GIST (19) (Table 1).

\section{Side effects}

The safety of ripretinib taken in a dosage of $150 \mathrm{mg}$ orally once daily was reported in the INVICTUS trial. Alopecia, myalgia, nausea, fatigue, palmar-plantar erythrodysesthesia and diarrhoe were the most common adverse events occurring in $\geq 20 \%$ of patients. In general, most of these adverse events were of grade 1 and 2 according to the Common Terminology criteria for adverse events version 4.03. In particular, no $>$ grade 2 hand-foot syndrome occurred in the study population. The most common adverse events rated as grade 3 or 4 consisted of lipase increase, hypertension and hypophosphatemia. However, the incidence of them were rather low $(\leq 5 \%)$. Nine percent of the patients suffered from treatment-related serious adverse events of which one cardiac failure, death of unknown cause, dyspnoe and an upper gastrointestinal haemorrhage seems noteworthy. Dose reduction and study treatment discontinuation was necessary in $6 \%$ and $5 \%$ of the ripretinib arm, respectively (19).

Two side effects deserve special consideration, cardiac dysfunction and the risk of developing new primary cutaneous malignancy. In a pooled safety population analysis cardiac failure (including cardiac failure, acute left ventricular failure, diastolic dysfunction, and ventricular hypertrophy) occurred in 1.7\%, including Grade 3 adverse reactions in $1.1 \%$. Therefore, initial assessment of ejection fraction by echocardiogram or MUGA scan should be considered prior to initiating ripretinib and clearly in case of cardiac symptoms or insufficiency during treatment. A permanent discontinuation of therapy is advised in case of grade 3 or 4 left ventricular systolic dysfunction. Additionally, $4.7 \%$ and $7 \%$ of patients developed a cutaneous squamous cell carcinoma in the Invictus trial and based on a pooled analysis, respectively. Dermatologic evaluations at treatment start and routinely during treatment is recommended $(17,19)$.

\section{Quality of life (QoL)}

Importantly, QoL data was repeatedly assessed during therapy and reported in the INVICTUS trial as well. The physical and role functioning on the one hand side and the overall health on the other hand were assessed with the European Organisation for Research and Treatment of Cancer Quality of Life Questionnaire for Cancer 30item (EORTC QLQ-C30) and the EuroQol 5-Dimension 5-Level /EQ-5D-5L) EuroQol visual analogue scale (EQ-VAS), respectively. Both parameters, the role and physical functioning and the overall health could be maintained in the active treatment arm whereas a decrease was documented in the placebo arm. Although statistical significance was not formally tested due to the hierarchal testing design the results were deemed to be clinically relevant (19).

\section{Discussion}

Ripretinib represents the current standard systemic therapy for patients with advanced GIST who have received prior treatment with three or more kinase inhibitors, including imatinib. The drug earns this therapy status due to several factors. First, there is a high level of trial evidence due to 
the randomised phase 3 trial setting. Secondly, a statistically significant and clinically relevant benefit in terms of PFS was shown in this study and thirdly, ripretinib is associated with a favourable side effect profile. Another strength of this study is the analysis of QoL that could be maintained under ripretinib therapy.

Nevertheless, further scientific and clinical questions need to be answered in the future. What is the ideal line of therapy for ripretinib? What is the optimal dose of ripretinib? What is the relationship between mutation status and efficacy? What resistance mechanisms develop with this medication and how should we treat patients progressing on ripretinib?

Due to the unusual dual mechanism of action and good therapy tolerance, an earlier use of this drug could be promising. Recently, Janku et al. (20) presented the results of the Phase 1 dose escalation and expansion study assessing the efficacy and tolerability of ripretinib in different treatment lines after imatinib resistance in 142 patients. Interestingly, the median PFS was highest when given in $2^{\text {nd }}$ line with 11.0 months, 95\% CI $(3.5,22.1)$, decreased with each treatment line and was 5.5 months, $95 \%$ CI (2.1, 8.1 ) in the $\geq 4$ th line, respectively. Additionally, doubling the ripretinib dose to $150 \mathrm{mg}$ BID after tumor progression on $150 \mathrm{mg}$ QD was associated again with a median PFS between 3.3 and 5.6 months, hence, an additional clinical benefit. The dose escalation came along with an only slight and obviously manageable increase in toxicity. Pharmacokinetic and dynamic results will be reported separately. The INTRIGUE trial, a randomised phase 3 trial comparing ripretinib $150 \mathrm{mg}$ QD with sunitinib as second-line therapy, is currently ongoing (NCT03673501). The optimal dose and dosing management will be a matter of debate in future.

Overall survival corresponds to the strongest and most relevant endpoint. Due to the special statistical design, no statistically significant difference could be proven here. Nevertheless, the results seem to indicate a prolongation of overall survival. In addition, serially used TKIs seems to be associated with improved survival in advanced GIST (21).

Patients with all kinds of mutational status could participate in the Invictus trial, including KIT and PDGFR wild-type GIST. Importantly, performing biopsy sampling before study inclusion and after previous treatment lines was mandatory. Unfortunately, the analysis of the predictive value of the reported mutations is missing up to now. Nevertheless, the efficacy results support signs of broadbased effectiveness.
While we are eagerly awaiting further data on the optimal use of ripretinib we are very much looking forward to having broaden our treatment potpourri and to gather real-life experience in our daily clinics.

\section{Acknowledgments}

Funding: None.

\section{Footnote}

Provenance and Peer Review: This article was commissioned by the Editorial Office, Gastrointestinal Stromal Tumor. The article did not undergo external peer review.

Conflicts of Interest: The author has completed the ICMJE uniform disclosure form (available at https://dx.doi. org/10.21037/gist-21-3). AK serves as the unpaid editorial board member of Gastrointestinal Stromal Tumor from Dec 2020 to Nov 2022. AK received Honoraria for lectures from Bayer and Eli Lilly, travel support from Ipsen, Pharmamar and Amgen. AK is on Advisory Board of Roche and Lilly.

Ethical Statement: The author is accountable for all aspects of the work in ensuring that questions related to the accuracy or integrity of any part of the work are appropriately investigated and resolved.

Open Access Statement: This is an Open Access article distributed in accordance with the Creative Commons Attribution-NonCommercial-NoDerivs 4.0 International License (CC BY-NC-ND 4.0), which permits the noncommercial replication and distribution of the article with the strict proviso that no changes or edits are made and the original work is properly cited (including links to both the formal publication through the relevant DOI and the license). See: https://creativecommons.org/licenses/by-nc-nd/4.0/.

\section{References}

1. Miettinen M, Lasota J. Gastrointestinal stromal tumors-definition, clinical, histological, immunohistochemical, and molecular genetic features and differential diagnosis. Virchows Arch 2001;438:1-12.

2. Gatta G, van der Zwan JM, Casali PG, et al. Rare cancers are not so rare: the rare cancer burden in Europe. Eur J Cancer 2011;47:2493-511. 
3. Miettinen M, Lasota J. Gastrointestinal stromal tumors: review on morphology, molecular pathology, prognosis, and differential diagnosis. Arch Pathol Lab Med 2006;130:1466-78.

4. Joensuu H. Risk stratification of patients diagnosed with gastrointestinal stromal tumor. Hum Pathol 2008;39:1411-9.

5. Joensuu H, Wardelmann E, Sihto H, et al. Effect of KIT and PDGFRA Mutations on Survival in Patients With Gastrointestinal Stromal Tumors Treated With Adjuvant Imatinib: An Exploratory Analysis of a Randomized Clinical Trial. JAMA Oncol 2017;3:602-9.

6. Demetri GD, von Mehren M, Blanke CD, et al. Efficacy and safety of imatinib mesylate in advanced gastrointestinal stromal tumors. N Engl J Med 2002;347:472-80.

7. Demetri GD, van Oosterom AT, Garrett CR, et al. Efficacy and safety of sunitinib in patients with advanced gastrointestinal stromal tumour after failure of imatinib: a randomised controlled trial. Lancet 2006;368:1329-38.

8. Demetri GD, Reichardt P, Kang YK, et al. Efficacy and safety of regorafenib for advanced gastrointestinal stromal tumours after failure of imatinib and sunitinib (GRID): an international, multicentre, randomised, placebocontrolled, phase 3 trial. Lancet 2013;381:295-302.

9. Blanke CD, Demetri GD, von Mehren M, et al. Longterm results from a randomized phase II trial of standardversus higher-dose imatinib mesylate for patients with unresectable or metastatic gastrointestinal stromal tumors expressing KIT. J Clin Oncol 2008;26:620-5.

10. Ganjoo KN, Villalobos VM, Kamaya A, et al. A multicenter phase II study of pazopanib in patients with advanced gastrointestinal stromal tumors (GIST) following failure of at least imatinib and sunitinib. Ann Oncol 2014;25:236-40.

11. Blay JY, Shen L, Kang YK, et al. Nilotinib versus imatinib as first-line therapy for patients with unresectable or metastatic gastrointestinal stromal tumours (ENESTg1): a randomised phase 3 trial. Lancet Oncol 2015;16:550-60.

12. Kang YK, Ryu MH, Ryoo BY, et al. Randomized phase III trial of imatinib (IM) rechallenge versus placebo (PL) in patients (pts) with metastatic and/or unresectable

doi: 10.21037 /gist-21-3

Cite this article as: Kollár A. Ripretinib—a new star in the firmament. Gastrointest Stromal Tumor 2021;4:2. gastrointestinal stromal tumor (GIST) after failure of at least both IM and sunitinib (SU): RIGHT study. J Clin Oncol 2013;31:abstr LBA10502.

13. Gebreyohannes YK, Schöffski P, Van Looy T, et al. Cabozantinib Is Active against Human Gastrointestinal Stromal Tumor Xenografts Carrying Different KIT Mutations. Mol Cancer Ther 2016;15:2845-52.

14. Schneeweiss M, Peter B, Bibi S, et al. The KIT and PDGFRA switch-control inhibitor DCC-2618 blocks growth and survival of multiple neoplastic cell types in advanced mastocytosis. Haematologica 2018;103:799-809.

15. Smith BD, Kaufman MD, Lu WP, et al. Ripretinib (DCC2618) Is a Switch Control Kinase Inhibitor of a Broad Spectrum of Oncogenic and Drug-Resistant KIT and PDGFRA Variants. Cancer Cell 2019;35:738-751.e9.

16. Villanueva MT. Ripretinib turns off the switch in GIST. Nat Rev Drug Discov 2019;18:499.

17. FDA Approved Drug Products: QINLOCK (ripretinib) tablets, for oral use. Available online: https://www.accessdata.fda.gov/drugsatfda_docs/ label/2020/213973s000lbl.pdf

18. George S, Heinrich M, Chi P et al. Initial Results of Phase 1 Study of DCC-2618, a Broad-spectrum KIT and PDGFRa Inhibitor, in Patients (pts) with Gastrointestinal Stromal Tumor (GIST) by Number of Prior Regimens. Ann Oncol 2018;29:viii576-95.

19. Blay JY, Serrano C, Heinrich MC, et al. Ripretinib in patients with advanced gastrointestinal stromal tumours (INVICTUS): a double-blind, randomised, placebocontrolled, phase 3 trial. Lancet Oncol 2020;21:923-34.

20. Janku F, Chi P, Heinrich M, et al. Ripretinib Intra patient Dose Escalation (IPDE) Following Disease Progression Provides Clinically Meaningful progression Free Survival (PFS) in Gastrointestinal Stromal Tumor (GIST) in Phase 1 Study. Ann Oncol;31:S914-33.

21. Call JW, Wang Y, Montoya D, et al. Survival in advanced GIST has improved over time and correlates with increased access to post-imatinib tyrosine kinase inhibitors: results from Life Raft Group Registry. Clin Sarcoma Res 2019;9:4. 